\title{
Effectiveness of mesh hernioplasty in incarcerated inguinal hernias
}

\author{
Georges Kamtoh, Radoslaw Pach, Wojciech Kibil, Andrzej Matyja, Rafal Solecki, Bartlomiej Banas, Jan Kulig \\ $1^{\text {st }}$ Department of General Surgery, Jagiellonian University Medical College, Krakow, Poland
}

Videosurgery Miniinv 2014; 9 (3): 415-419

DOI: $10.5114 /$ wiitm.2014.43080

\begin{abstract}
Introduction: The use of mesh is still controversial in patients undergoing emergency incarcerated hernia repair, mostly because of potential infectious complications.

Aim: The main aim of this study was to assess the efficacy of tension-free methods in treating incarcerated inguinal hernias (IIH), with and without intestine resection. The secondary aim was to establish an algorithm on how to proceed with incarcerated hernias.

Material and methods: A retrospective analysis of patients who underwent surgery due to an inguinal hernia at the First Department of General Surgery Jagiellonian University Medical College in Krakow, in the period 1999-2009. Operative methods included Lichtenstein, Robbins-Rutkow and Prolene Hernia System. The rate of postoperative complications was compared in patients who underwent elective and emergency surgery.

Results: The study group consisted of 567 patients (546 male) age 19-91 years. In this group 624 hernias were treated using the three tension-free techniques - 295 using the Lichtenstein method, 236 using PHS and 93 using the $R R$ technique. Out of the 561 operations $89.9 \%$ were elective. No correlation $(p>0.05)$ was found between the type of surgery and such complications as postoperative pain duration and intensity, fever, micturation disorders, wound healing disorders, testicle hydrocoele, testicle atrophy, spermatic cord cyst, sexual dysfunction, wound dehiscence, wound suppuration, seroma, haematoma and hernia recurrence.

Conclusions: Mesh repairs can be safely performed while operating due to an IIH. The use of a synthetic implant, in emergency IIH repairs, does not increase the rate of local complications. Synchronous, partial resection of the small intestine, due to intestinal necrosis, is not a contraindication to use mesh.
\end{abstract}

Key words: mesh repair, incarcerated inguinal hernias, tension-free.

\section{Introduction}

The only definite treatment for inguinal hernia is surgery. In the case of elective surgery, the treatment of choice is the use of a synthetic implant. However, in everyday clinical practice, it is still possible to meet patients who, because of negligence or late diagnostics, suffer from an incarcerated hernia. The use of mesh is still controversial in patients undergoing emergency incarcerated hernia repair, mostly because of infectious complications [1-3].

\begin{abstract}
Aim
The main aim of this study was to assess the efficacy of tension-free methods in treating incarcerated inguinal hernias, with and without intestine resection. The secondary aim was to establish an algorithm on how to proceed with incarcerated hernias, using the three most common tension-free hernia repair methods: Lichtenstein, Robbins-Rutkow (RR) and the Prolene Hernia System (PHS).
\end{abstract}




\section{Material and methods}

To be included in the analysis a patient had to be operated on due to an incarcerated inguinal hernia in the First Department of General, Oncological and Gastrointestinal Surgery (Jagiellonian University Medical College - JUMC) in Krakow, in the period 1999-2009. Operative methods included in the analysis were Lichtenstein, RR and PHS. To assess the efficacy of the above-mentioned methods, in treating incarcerated inguinal hernias, the patients were divided into those who underwent elective and emergency surgery. The rate of postoperative complications was compared between groups.

Patients undergoing emergency surgery due to hernia incarceration, or with risk factors, preoperatively received antibiotics.

After being discharged home, patients underwent regular check-ups at the Outpatient Clinic of the First Department of General, Oncological and Gastrointestinal Surgery JUMC. The control visits were scheduled 10 days after surgery, next every 3 months during the first postoperative year, then every 6 months, or with a higher frequency, in case of any postoperative complaints. This did not relate to patients with postoperative complications, who were consulted more often, depending on the indications.

The clinic has its own computer system, where all patient data (whether hospital or ambulatory) are gathered, including medical history (complaints, family history, smoking/drinking, concomitant diseases, medications taken, past illnesses, previous operations), physical examination, diagnostic imaging, course of hospitalization, surgical protocol (date, duration of surgery, type of surgery, type of anaesthesia, name of the personnel performing the surgery) and discharge documents.

The analysis was conducted based on data gathered from the medical history of each patient (gender, age, weight, height, type of hernia according to Nyhus classification, duration of the surgery and hospitalization). The analysis included the occurrence of different surgical complications (short- and long-term), comparing emergency versus elective procedures.

\section{Results}

The study group consisted of 567 patients (546 male and 21 female) aged between 19 and 91 years.
In this group 624 hernias were treated using the three tension-free techniques - 295 using the Lichtenstein method, 236 using PHS and 93 using the Robbins-Rutkow technique. Among the procedures 561 were elective (89.9\%) and 63 were emergency $(10.1 \%)$. Out of the incarcerated hernias $52.4 \%$ were right-sided and $47.6 \%$ were left-sided. Bilateral incarcerated inguinal hernia repair was performed in only 2 cases.

Among women 20 procedures (90.9\%) were elective and 2 (9.1\%) were emergency, while in men 541 (89.9\%) were elective and 61 (10.1\%) were emergency. Thus no statistically significant difference was noted $(p>0.05)$.

When considering the Lichtenstein method, there were 248 elective procedures (44.2\% of all elective procedures) and 47 emergency procedures (74.6\% of all emergency procedures). Respectively, while performing the RR method, these numbers were 84 (15\%) and 9 (14.3\%), and using the PHS method they were 229 (40.8\%) and 7 (11.1\%). Emergency procedures were more often performed using the Lichtenstein method $(p<0.0001)$.

When analysing different age groups $(<35,35-65$ and $>65$ ) it was noted that emergency procedures used more often in people over 65 years of age $(p=0.001)$.

Body mass did not correlate with the type of surgery performed $(p>0.05)$. The mean surgery duration for elective procedures was 58.74 min (median 55.0), and for emergency procedures was $88.24 \mathrm{~min}$ (median 85.0). Emergency procedures lasted significantly longer then the elective ones $(p<0.0001)$.

The mean time of hospitalization after elective procedures was 2.29 days (median 1.0), and after emergency procedures 4.19 days (median 4.0). The time of hospitalization was statistically shorter after elective procedures $(p<0.0001)$.

Postoperative pain duration was assessed on the day of the surgery, as well as 7, 30, 90 and above 90 days postoperatively. No correlation was found between the type of surgery performed and the duration of postoperative pain $(p=0.159)$. When analysing patients with chronic pain (>90 days) and their pain intensity (VAS), no correlation was noted with the type of surgery performed ( $p=1.000$ and $p=0.073$ respectively).

No correlation was also found between the type of surgery and other complications (Table I) such as fever $(p=0.305)$, micturation disorders $(p=0.804)$, 
Table I. Number of complications depending on the type of surgery (elective vs. emergency)

\begin{tabular}{|lccc|}
\hline Complications & Elective surgery & Emergency surgery & Value of $p$ \\
\hline Recurrence & 11 & 2 & NS \\
\hline Chronic pain & 17 & 1 & NS \\
\hline Intensity of chronic pain (0-10 VAS) & 2 & 0.073 (NS) & NS \\
\hline Suppuration & 10 & 2 & NS \\
\hline Wound inflammation & 24 & 1 & NS \\
\hline Seroma & 38 & 3 & NS \\
\hline Hematoma & 50 & 12 & NS \\
\hline Scrotum/pudendal lip edema & 42 & 5 & NS \\
\hline Micturation disorders & 10 & 1 & NS \\
\hline Testicle hydrocoele & 3 & 0 & NS \\
\hline Testicle atrophy & 1 & 0 & NS \\
\hline Spermatic cord cyst & 20 & 2 & NS \\
\hline Wound healing disorders & 14 & 1 & NS \\
\hline Sexual dysfunction & 1 & 0 & \\
\hline Death & & & \\
\hline
\end{tabular}

NS - non significant $(p>0.05)$

wound healing disorders $(p=1.000)$, testicle hydrocoele $(p=1.000)$, testicle atrophy $(p=1.000)$, spermatic cord cyst $(p=1.000)$, sexual dysfunction $(p=1.000)$, wound dehiscence $(p=1.000)$, wound suppuration (1.6\% of elective and $1.6 \%$ of emergency procedures; $p=1.000$ ), seroma ( $4.3 \%$ of elective and $1.6 \%$ of emergency procedures; $p=0.499$ ), haematoma (6.8\% elective and $4.8 \%$ emergency procedures; $p=0.788)$ and hernia recurrence $(p=0.643)$.

Only scrotum/pudendal lip oedema occurred more often after emergency procedures $(p=0.020)$. This complication occurred after $8.9 \%$ of elective and $19 \%$ of emergency procedures. On the other hand, $80 \%$ of patients with scrotum/pudendal lip oedema were operated electively and only $19.4 \%$ on emergency.

Only 2 elective patients had to undergo reoperations. One patients died in the postoperative period. He underwent surgery without intestine resection. This incident was not directly associated with the surgery itself.

Among patients undergoing emergency operations, a synchronous intestine resection was performed in 8 cases (12.69\% of elective procedures). This pertained only to men with a unilateral, right- sided inguinal hernia. In 6 of those cases the Lichtenstein method was used, and in 2 the PHS $(p=0.418)$. No correlation was noted between intestine resection and patient death $(p=0.711)$, micturation disorders $(p=0.310)$, wound healing disorders $(p=0.537)$, testicle hydrocoele $(p=0.666)$, sexual dysfunction $(p=0.650)$, chronic pain and its intensity ( $p=0.477$ and $p=0.051$ respectively), seroma $(p=0.711)$, haematoma $(p=0.518)$, scrotum $/ \mathrm{pu}$ dendal lip oedema $(p=0.177)$, and wound inflammation or suppuration $(p=0.599)$.

\section{Discussion}

Similarly, as in our material, the literature data show that hernia incarceration occurs in $8 \%$ to $15 \%$ of patients with inguinal hernias [4-9]. The mean age of those patients is usually above 60 years of age $[7,10,11]$.

The contents of a hernial sac are most often the loops of the small intestine $[1,10]$. This is associated with increased mobility of intestinal loops, which is additionally intensified by peristalsis. In a hernial $\mathrm{sac}$, one can sometime find the coecum as well as the vermiform appendix (0.5\% to $5 \%$ of cases) [12]. 
The mortality rate after an incarcerated hernia repair varies from $2.6 \%$ to $11 \%[5,10,11,13]$. This is most often so in cases needing intestine resection [14]. According to the authors' opinion, early detection and surgical treatment is crucial [15]. Other factors that also increase the mortality rate are older age and serious concomitant diseases [13]. The reason for the low mortality rate obtained in our study is probably the fact of early qualification for surgery and strict postoperative patient observation.

Infection and wound suppuration are among the most common surgical complications occurring after inguinal hernia surgery. This is especially dangerous in cases when deep abscesses develop around the mesh. Thus, using meshes in a potentially infected operating field, such as in the case of an incarcerated inguinal hernia, is still controversial. The type of the mesh used has an important influence on the occurrence of infection. D'Ambrosio et al. [16], in their study assessing the efficacy of polypropylene mesh in treating inguinal hernias, detected a beneficial influence of the polypropylene mesh structure on the rate of infectious complications. This is associated with the large diameter of pores (> 70 microns), which allows the macrophages and granulocytes to contact the bacteria. Additionally, when compared with a PTFE mesh, the use of a polypropylene mesh allows faster scar tissue formation. In contrast, the study by Pielaciński et al. [17] showed no difference between heavyweight non-absorbable polypropylene mesh and lightweight partially absorbable mesh in terms of early postoperative complications.

According to the authors, the polypropylene meshes can be safely used in inguinal hernia surgery, even when intestine resection is needed [16]. In the analysed material, operations were performed only with the use of polypropylene meshes. This might explain the low percentage of infectious complications, and the lack of necessity to remove the meshes in case of wound suppuration. Our results correlate with other literature data, where the occurrence of wound suppuration does not differ between emergency and elective procedures using synthetic implants [18]. This also relates to other short- and long-term complications such as wound healing disorders, testicle hydrocoele, spermatic cord cyst, sexual dysfunction, seroma, haematoma and chronic pain [19]. Some authors reported that early complications are more frequently observed in patients with giant hernias (in whom the hernia sac is below the point located on the inner area of the thigh, in the middle of its length when a patient is in a standing position) so they are associated with size of hernia but not with type of synthetic mesh [20].

Incarcerated hernias are the second most common reason for small intestine obstruction. If intestine necrosis occurs, this may significantly worsen the prognosis of patients with incarcerated hernias. The time of hernia incarceration influences the vitality of the intestine. If incarceration lasts for more than $6 \mathrm{~h}$, then this is considered the main factor associated with the need to perform resection. This time is closely correlated with postoperative mortality [21]. About $15 \%$ of patients with an incarcerated inguinal hernia require intestine resection due to necrosis [22]. In our material, plastic inguinal hernia repair in case of intestine resection did not increase the complication rate. Even when taking into account the relatively small study group, the obtained results support the view that mesh implantation can be successfully performed during emergency procedures, even when intestine resection is needed. This is confirmed in the works of other authors [ 6 , $23,24]$. This is especially important in the case of necrosis without intestine perforation, peritonitis, intra-abdominal abscess or the need to resect the large intestine [4, 5]. The main reason for outcome worsening, when treating incarcerated inguinal hernias, is intestine necrosis with accompanying peritonitis, and not just the fact of using mesh [5]. The next reason supporting the use of mesh in emergency procedures is the lower rate of hernia recurrence. According to the authors, after emergency procedures performed using tension techniques, the recurrence rate is around $40 \%$, while when using tension-free techniques this rate varies from $1 \%$ to $22 \%$ $[5,25,26]$. In a study comparing methods of plastic incarcerated inguinal hernia repair, with and without the use of mesh, the authors observed a lower recurrence rate in patients operated on with the Lichtenstein technique (tension-free) [26].

The use of antibiotic prophylaxis is still a subject of many controversies. However, due to surgery type (elective vs. emergency) and the risk of intestine resection (thus operating in an infected field), the use of antibiotic prophylaxis is recommended. This is especially important in patients with risk factors such as diabetes, chronic obstructive pulmonary disease, immunological disorders, and during glucocorticosteroid therapy [13]. Older age (above 65 years) is 
also a negative prognostic factor in patients with an incarcerated inguinal hernia $[27,28]$. This is especially so in patients with many concomitant diseases [6]. It is assumed that, apart from strictly following the rules of aseptic and antiseptic behaviour, systematic antibiotic prophylaxis allowed a low wound infection rate to be obtained in this study.

\section{Conclusions}

Mesh repairs can be safely performed when operating due to an incarcerated inguinal hernia. The use of a synthetic implant, in emergency inguinal hernia repairs, does not increase the rate of local complications. It seems that synchronous, partial resection of the small intestine, due to intestinal necrosis, is not a contraindication to use mesh.

\section{References}

1. Henry X, Randramananstoa V, Verhaeghe P, et al. Le materiel prothétique a t-ilune place raisonnabledans le traitement des urgencies herniaires? Chirurgie 1995; 120: 123-8.

2. Stoppa RE. The treatment of complicated groin and incisional hernias. World J Surg 1989; 64: 269-85.

3. Elsebae MM, Nasr M, Said M. Tension-free repair versus Bassini technique for strangulated inguinal hernia: a controlled randomized study. Int J Surg 2008; 6: 302-5.

4. Ueda J, Nomura T, Sasaki J, et al. Prosthetic repair of an incarcerated groin hernia with small intestinal resection. Surg Today 2012; 42: 359-62.

5. Derici H, Unalp HR, Nazli O, et al. Prosthetic repair of incarcerated inguinal hernias: is it a reliable method? Langenbecks Arch Surg 2010; 395: 575-9.

6. Atila K, Guler S, Inal A, et al. Prosthetic repair of acutely incarcerated groin hernias: a prospective clinical observational cohort study. Langenbecks Arch Surg 2010; 395: 563-8.

7. Adesunkanmi AR, Badmos TA, Salako AA, et al. Groin hernias in patients 50 years of age and above pattern and outcome of management in 250 consecutive patients. West Afr J Med 2000; 19: 142-7.

8. Kulah B, Duzgun AP, Moran M, et al. Emergency hernia repairs in elderly patients. Am J Surg 2001; 182: 455-9.

9. Kurt N, Oncel M, Ozkan Z, et al. Risk and outcome of bowel resection in patients with incarcerated groin hernias: retrospective study. World I Surg 2003; 27: 741-3.

10. Palot JP, Flament JB, Avisse C, et al. Utilisation des prothèsesdans les conditions de la chirurgied'urgences. Etude rétrospective de 204 hernies de l'aineétranglées. Chirurgie 1996; 121: 48-50.

11. Alvarez JA, Baldonedo RF, Bear IG, et al. Incarcerated groin hernias in adults: presentation and outcome. Hernia 2004; 8: 121-6.

12. Gurer A, Ozdogan M, Ozlem N, et al. Uncommon content in groin hernia sac. Hernia 2006; 10: 152-5.
13. Kulah B, Kulacoglu IH, Oruc MT, et al. Presentation and outcome of incarcerated external hernias in adults. Am J Surg 2001; 181: 101-4.

14. Nisson H, Stylianidis G, Haapamäki M et al. Mortality after groin hernia surgery. Ann Surg 2007; 245: 656-60.

15. Giustetto A, Zan S, Sacchetti M, et al. The surgical treatment of strangulated inguinalcrural hernias in geriatric patients. Minerva Chir 1994; 49: 1275-80.

16. D'Ambrosio R, Capasso L, Sgueglia S, et al. The meshes of polypropylene in emergency surgery for strangulated hernias and incisional hernias. Ann Ital Chir 2004; 75: 569-73.

17. Pielaciński K, Szczepanik AB, Wróblewski T. Effect of mesh type, surgeon and selected patients' characteristics on the treatment of inguinal hernia with the Lichtenstein technique. Randomized trial. Videosurgery Miniinv 2013; 8: 99-106.

18. Bessa SS, Katri KM, Abdel-Salam WN, et al. Early results from the use of the Lichtenstein repair in the management of strangulated groin hernia. Hernia 2007; 11: 239-42.

19. Lohsiriwat V, Sridermma W, Akaraviputh T, et al. Surgical outcomes of Lichtenstein tension-free hernioplasty for acutely incarcerated inguinal hernia. Surg Today 2007; 37: 212-4.

20. Bierca J, Kosim A, Kołodziejczak M, et al. Effectiveness of Lichtenstein repairs in planned treatment of giant inguinal hernia - own experience. Videosurgery Miniinv 2013; 8: 36-42.

21. Nilsson $\mathrm{H}$, Nilsson $\mathrm{E}$, Angerås $\mathrm{U}$, et al. Mortality after groin hernia surgery: delay of treatment and cause of death. Hernia 2011; 15: 301-7.

22. Brasso K, Nielsen KL, Christiansen J. Long-term results of surgery for incarcerated groin hernia. Acta Chir Scand 1989; 155: 583-5.

23. Wysocki A, Kulawik J, Pozniczek M, et al. Is the Lichtenstein operation of strangulated groin hernia a safe procedure? World J Surg 2006; 30: 2065-70.

24. Papaziogas B, Lazaridis C, Makris J, et al. Tension-free repair versus modified Bassini technique (Andrews technique) for strangulated inguinal hernia: a comparative study. Hernia 2005; 9: 156-9.

25. Melis P, van der Drift DG, Sybrandy R, et al. High recurrence rate 12 years after primary inguinal hernia repair. Eur J Surg 2000; 166: 313-4.

26. Haapaniemi S, Nilsson E. Recurrence and pain three years after groin hernia repair. Validation of postal questionnaire and selective physical examination as a method of follow-up. Eur J Surg 2002; 168: 22-8.

27. Bueno Lledó J, Sosa Quesada Y, Gomez I Gavara, et al. Prosthetic infection after hernioplasty. Five years' experience. Cir Esp 2009; 85: 158-64.

28. Andrews NJ Presentation and outcome of strangulated external hernia in a district general hospital. Br J Surg 1981; 68: 329-32.

Received: 30.12.2013, accepted: 17.02.2014. 